\title{
Longwing (Heliconius) butterflies combine a restricted set of pigmentary and structural coloration mechanisms
}

\author{
Bodo D. Wilts ${ }^{12^{*}}$ (D, Aidan J. M. Vey ${ }^{1}$, Adriana D. Briscoe ${ }^{3}$ and Doekele G. Stavenga ${ }^{1}$
}

\begin{abstract}
Background: Longwing butterflies, Heliconius sp., also called heliconians, are striking examples of diversity and mimicry in butterflies. Heliconians feature strongly colored patterns on their wings, arising from wing scales colored by pigments and/or nanostructures, which serve as an aposematic signal.

Results: Here, we investigate the coloration mechanisms among several species of Heliconius by applying scanning electron microscopy, (micro)spectrophotometry, and imaging scatterometry. We identify seven kinds of colored scales within Heliconius whose coloration is derived from pigments, nanostructures or both. In yellow-, orange- and red-colored wing patches, both cover and ground scales contain wavelength-selective absorbing pigments, 3-OH-kynurenine, xanthommatin and/or dihydroxanthommatin. In blue wing patches, the cover scales are blue either due to interference of light in the thin-film lower lamina (e.g., H. doris) or in the multilayered lamellae in the scale ridges (so-called ridge reflectors, e.g., H. sara and H. erato); the underlying ground scales are black. In the white wing patches, both cover and ground scales are blue due to their thin-film lower lamina, but because they are stacked upon each other and at the wing substrate, a faint bluish to white color results. Lastly, green wing patches (H. doris) have cover scales with blue-reflecting thin films and short-wavelength absorbing 3-OH-kynurenine, together causing a green color.
\end{abstract}

Conclusions: The pigmentary and structural traits are discussed in relation to their phylogenetic distribution and the evolution of vision in this highly interesting clade of butterflies.

Keywords: Light scattering, Signaling, Thin films, Nymphalidae, Ommochromes, Aposematism, Butterflies

\section{Background}

The colorful wings of butterflies have long caught the eye of natural biologists [1]. Butterfly wing coloration patterns have numerous functions, including mate attraction and identification, concealment, and warning (aposematic) signaling [2-4]. Because wing coloration often plays multiple functions simultaneously [5], a possible conflict exists between the expression of the different ways of signaling, which can be overcome by spatially or dynamically separating the color signals. For instance, the coloration of the ventral wing side is commonly involved in predator avoidance (e.g. by camouflage),

\footnotetext{
*Correspondence: bodo.wilts@unifr.ch

${ }^{1}$ Computational Physics, Zernike Institute for Advanced Materials, University

of Groningen, Nijenborgh 4, NL-9747AG Groningen, The Netherlands

${ }^{2}$ Adolphe Merkle Institute, University of Fribourg, Chemin des Verdiers 4,

$\mathrm{CH}-1700$ Fribourg, Switzerland

Full list of author information is available at the end of the article
}

since most butterflies fold their wings above the body when at rest, so that only the ventral wing sides are visible [5-7]. The often brightly-colored dorsal wing sides predominantly function in intra-specific communication, which however can also make the butterflies highly visible to predators. Aposematic species, displaying visible warning colors [2, 3], are often involved in complex mimicry 'rings', where multiple species converge on a similar coloration pattern $[3,4,8,9]$. Such mimicry has clear fitness advantages, as the participating species all benefit from looking alike. However, in order to successfully reproduce, members of species involved in mimicry rings must be able to distinguish conspecifics from heterospecifics [10, 11].

The mechanisms underlying coloration are usually distinguished in having a physical or chemical basis. Physical colors are created by orderly arranged nanostructures, and chemical colors are due to wavelength-selective absorbing pigments. The various physical and chemical mechanisms 
contributing to butterfly wing coloration are often considered to operate separately from each other [12], but sometimes pigmentary and structural coloration combine non-trivially [13-15]. For example, in the wings of cabbage whites (pierids), the UV-absorbing pigment leucopterin, causing low wing reflectance in the ultraviolet, is located in densely packed, ellipsoidal-shaped beads in the wing scales that serve to enhance the scattering in the (for humans) visible wavelength range, resulting in brilliant white wings $[14,16,17]$. Birdwing butterflies (Ornithoptera) have extremely colorful wings due to specially-structured wing scales, which contain papiliochrome pigments acting as a spectral filter on a chirped multilayer reflector, thus tuning the scale color [15]. In the nymphaline peacock butterfly, Aglais io, the cover scales in the blue eyespots at the dorsal wings are blue due to the lower lamina acting as a blue-reflecting thin film [18]. The ground scales in the eyespots are black, because of highly concentrated melanin, thus serving as a contrast enhancing background. However, when the ground scales have the same structure as the blue cover scales, the stack of scales together with the wing substrate create a faint-bluish or even whitish color [18].

Here, we investigate the spectral properties of several Heliconius species in order to identify the main pigmentary and structural mechanisms that determine the wing coloration patterns. Heliconians present an attractive study system, because the coloration of many species is bright and simple-patterned. Furthermore, the genetic processes that determine the wing coloration and the role of color in the ecology of the species in this group have been well studied $[2,9,10,19,20]$. Heliconius species further present the prime example of aposematic coloration, rapid speciation and the presence of Müllerian mimicry rings [2, 21-23]. To elucidate the spectral properties of heliconian butterfly wing scales, we applied various optical methods as well as electron microscopy. We place the study in a phylogenetic context in order to demonstrate how some coloration mechanisms are restricted to certain taxa, while others are widespread. We highlight the value of using reflectance spectrometry to quantify and distinguish the spectral properties of butterfly wings.

\section{Methods}

\section{Specimen}

We investigated 12 Heliconius species (and a number of subspecies) (Additional file 1: Table S1) representing $\sim 25 \%$ of species in the genus [24]. Specimens were either ordered from commercial suppliers (dried specimen were obtained from Tropical Butterflies and Insects of America, Tampa, FL, USA and Worldwide Butterflies, Dorset, UK; pupae of $H$. doris were obtained from Costa
Rica Entomological Supply, Costa Rica) or were from the collection of the National Museum of Natural History Naturalis, Leiden, the Netherlands. Photographs of pinned specimens were taken with a Nikon D70 equipped with an F70 macro objective lens and a Nikon SB-800 ring flash.

\section{Scanning electron microscopy}

The morphological structure of wing scales was visualized with a Tescan Mira 3 field-emission scanning electron microscope (SEM), using samples sputtered with a $\sim 3 \mathrm{~nm}$ thick layer of platinum/palladium (80:20 wt\%).

\section{Imaging scatterometry}

We applied imaging scatterometry to visualize the farfield angular distribution of the light scattered from single scales (2-5 scales per specimen), glued at the end of pulled glass micropipettes $[25,26]$. The sample was positioned in the first focal point of the scatterometer's ellipsoidal mirror, which collects light from a full hemisphere. A xenon lamp was used for illumination, producing a narrow aperture beam $\left(5^{\circ}\right)$ and a small spot size (diameter $\sim 30 \mu \mathrm{m}$ ). A piece of magnesium oxide served as a white diffuse reference object. Scatterogram images were acquired by an Olympus DP70 camera and were subsequently corrected for geometrical distortions using a Matlab routine.

\section{Spectrometry}

Reflectance spectra of intact wings (average of 2-5 individual spectra of all 12 investigated species) were measured with a bifurcated probe linked to a halogen/ deuterium light source and an Avantes AvaSpec-2048-2 (Avantes, Apeldoorn, NL) CCD detector array spectrometer. The angle of illumination was about normal with respect to the wing surface. A microspectrophotometer (MSP), consisting of a Leitz Ortholux microscope and the Avantes spectrometer, was used to measure reflectance spectra from both sides of single scales (abwing upper side; adwing - lower side), which were removed from the wing and glued to the tip of pulled glass pipettes. A white diffuse standard (Avantes WS-2) was used as a reference for all reflectance measurements. Absorbance spectra of single wing scales were obtained with the MSP by measuring the transmittance of single scales embedded in immersion fluid (Cargille Labs, Cedar Grove, NJ, USA; Series A) with a refractive index of 1.56 , closely matching the refractive index of chitin [27].

\section{Pigment extraction and absorbance spectra}

Pigments from the red, orange and yellow scales on the wings were extracted from a total of 1 individual per species in a solution of 50:1 methanol: $1 \mathrm{M}$ hydrochloric 
acid. Wing fragments excised from colored patches were placed in $600 \mu \mathrm{l}$ of this solution in Eppendorf tubes for $\sim 1 \mathrm{~h}$. Solutions were then centrifuged for $4 \mathrm{~min}$ at $14000 \mathrm{rpm}$ (Eppendorf centrifuge 5418), and the supernatant removed for liquid spectrometry. A liquid UV-VIS spectrometer (Perkin Elmer LAMBDA 365) was used to measure the absorbance spectra of the pigment in solution against a reference of pure $\mathrm{MeOH}: \mathrm{HCl}$ solution. All chemicals $(\mathrm{MeOH}$ and $\mathrm{HCl})$ were purchased from Sigma Aldrich and used as received.

\section{Ancestral state reconstruction of wing pigmentary and structural coloration}

The Bayesian phylogeny of Kozak et al. [24] including branch lengths was used to trace the evolution of two types of scales that derive their color primarily from pigmentation [i) 3-OHK (yellow), ii) xanthommatin/dihydroxanthommatin (orange/red)], three types of scale that derive their color solely from their structure [iii) simple blue scales (blue), iv) simple blue scales stacked (white), v) multi-ridge reflectors coloration (blue)], and one type of scale whose color derives from both [vi) simple blue scales containing 3-OHK (green)]. A total of sixteen Heliconius species/subspecies with representatives of all seven major clades and one outgroup species in the genus Eueides were included (Additional file 1: Table S1). Species entirely missing any particular trait were coded as 0 , species with reflectance spectra characteristic of a certain trait were coded as 1 . Ancestral state reconstruction was performed in Mesquite (ver. 3.2; ref. [28]) using the maximum likelihood option with default settings.

\section{Results}

\section{Colors and reflectance spectra of longwing butterflies}

The wings of Heliconius butterflies are well-known for their striking wing patterns that feature bright yellow, orange or red-colored patches in a velvet black frame. This frame may show metallic blue patches in some species. The butterflies have a total wingspan of 4-6 cm and the hindwings are much smaller than the forewings, resulting in a highly asymmetrical appearance characteristic for heliconians.

Figure 1 presents four Heliconius species from four of the seven major clades within the genus [24]. $H$. telesiphe and $H$. melpomene have dorsal forewings with prominent red wing patches, and dorsal hindwings with white (H. telesiphe) or yellow (H. melpomene) bands (Fig. 1a, b). H. doris and $H$. sara feature prominent yellow wing patches combined with bluish wing areas (Fig. 1d, e). To investigate the nature of the variously colored wing areas, we measured the reflectance spectra of these areas with a bifurcated optical probe (Fig. 1c, f). The reflectance spectra of the yellow- and red-colored areas (Fig. 1c, \#1-4) are characteristic of pigmentary (or chemical) colored scales that absorb incident light in a restricted wavelength range $[18,29,30]$. The reflectance spectra of the velvet black wing areas (Fig. 1c, \#5), found in all investigated heliconian butterflies, are typical for melanized wing scales [18]. As we will further discuss below, the spectra of the white and blue wing areas (Fig. 1f, 6-8) are characteristic of structural (or physical) colored scales.

\section{Spatial and spectral reflection properties of pigmentary- colored scales}

In our analysis of the spatial and spectral reflection properties of the scales, we first focused on the pigmented wing scales. Figure 2a-c presents the case of a red wing scale of $H$. telesiphe. Scanning electron microscopy (SEM) confirmed that the scale has the classic nymphalid ultrastructure [18, 31, 32]. A set of parallel ridges, connected by crossribs, forming together the upper lamina, is mounted on an approximately flat lower lamina (Fig. 2a). Contrary to Gilbert et al. [32], we observe that all pigmented scales feature the same ultrastructure (see Additional file 1: Figure S1).

Figure $2 \mathrm{~b}$ shows a scatterogram created by a narrowaperture, white-light beam illuminating a small area of the upper lamina of a red scale. The scatterogram demonstrates that the red part of the white incident light is back-scattered into the full hemisphere (Fig. 2b). This is a characteristic feature of pigmentary-colored scales, where diffuse scattered light is spectrally-filtered by a short-wavelength-selective absorbing pigment [33]. The additional, weak-whitish line in the scatterogram, which is perpendicular to the ridges, shows that the set of parallel ridges acts as a diffraction grating (Fig. 2b); for similar cases see $[18,25,30,34]$.

Rotating the same scale by $180^{\circ}$, that is, illuminating the scale's underside, yielded a scatterogram with a strongly-directional, bluish reflection and a muchreduced diffuse red scattering (Fig. 2c). We interpret this as that the lower lamina acts as an approximately flat, blue-reflecting thin film $[18,35]$. Since the lower lamina's reflection is low in the longer wavelength range, the red transmitted light will be scattered by the upper lamina, which thus contributes the relative weak, diffuse red scattering in Fig. 2c.

We note that the situation was fully opposite in the previous case of illumination, from the side of the upper lamina. Incident white light was then mainly scattered by the red-pigmented upper lamina, and the little shortwavelength light that reached (and was reflected by) the lower lamina, was again severely spectrally filtered by the upper lamina, resulting in a negligible contribution of short-wavelength light to the reflectance. To substantiate this interpretation, we measured the reflectance 


\section{a}

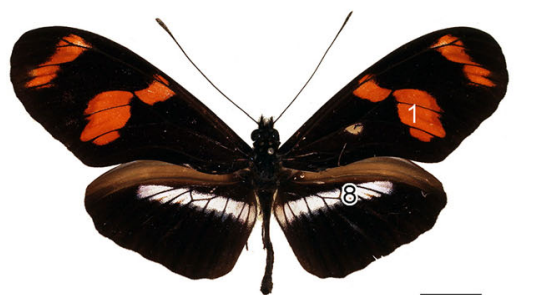

b

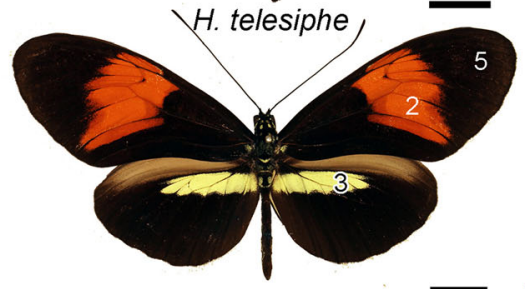

H. melpomene

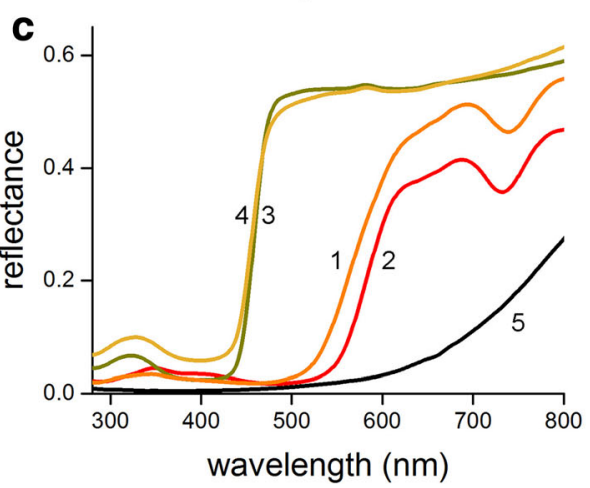

d

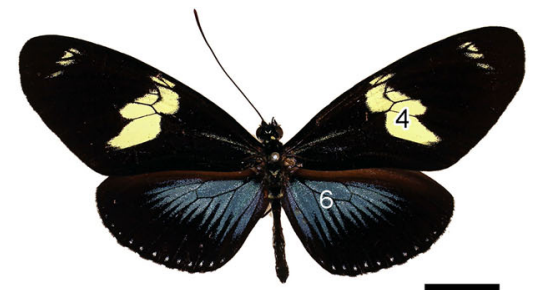

e

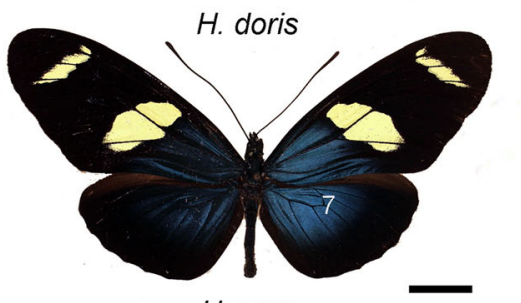

H. sara

$\mathbf{f}$

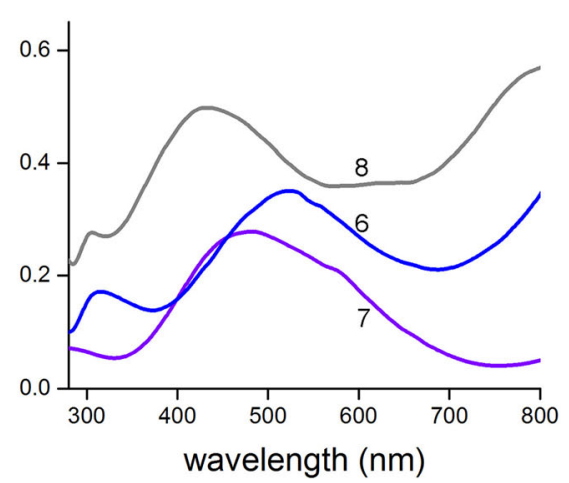

Fig. 1 Representative coloration of Heliconius species. a, b, d, e Photographs of pinned specimen of $H$. telesiphe, $H$. melpomene, $H$. doris, and H. sara, respectively. Scale bars: $1 \mathrm{~cm}$. c, f Reflectance spectra of the various colored dorsal wing areas measured with a bifurcated probe. The number near each spectrum corresponds to the wing location in panels a-d where the spectrum was measured

spectra of both sides of isolated, single scales with a microspectrophotometer (Fig. 2d). The reflectance of the abwing (upper) side of the red scale is very low at short wavelengths and high in the longer wavelength range, due to a pigment absorbing in the short-wavelength range. The reflectance of the adwing (lower) side has a major blue peak, indicating a thin film with thickness $\sim 210 \mathrm{~nm}$ (see refs. [18, 35] for more details on how to extract the film thickness from reflectance measurements). Although for an ideal thin film with thickness $210 \mathrm{~nm}$ the reflectance should be zero at $\sim 650 \mathrm{~nm}$, the red-light-scattering upper lamina clearly causes a nonnegligible contribution to the reflectance in the longer wavelength range. The spatial and spectral reflection properties of yellow scales were found to be basically similar as only the absorption is shifted to shorter wavelengths.

\section{Pigmentary basis of the coloration}

To further characterize the pigments in the pigmentarycolored scales, we performed microspectrophotometry on single wing scales of $H$. telesiphe as well as of several other Heliconius species (at least 2-5 wing scales per specimen; Fig. 2e, Additional file 1: Table S1). To suppress the distortions by light scattering in the measurements of the absorbance spectra, we immersed the scales in a fluid with refractive index $n=1.56$, closely matching that of cuticular chitin [27]. We found three pigments present in the scales: a yellow pigment with in situ peak absorption at $\sim 420 \mathrm{~nm}$, an orange pigment with peak absorption at $\sim 480 \mathrm{~nm}$, and a red pigment with peak absorption at $\sim 520 \mathrm{~nm}$ (Fig. 2e).

We further studied the wing scale pigmentation by exposing the scales to a solution of 50:1 methanol: $1 \mathrm{M}$ hydrochloric acid, which is known to dissolve ommochromes as well as their precursor 3-hydroxy-DL-kynurenine (3-OHK) [2]. The absorbance spectra of the obtained extractions, a yellow $(\mathrm{Y})$, orange $(\mathrm{O})$ and red (R) pigment (Fig. 2f), are highly similar to the in situ measurements (Fig. 2e), except for a distinct shift to shorter wavelengths of the extracted pigment spectra. This hypsochromic shift of $\sim 20-45 \mathrm{~nm}$ must be attributed to the different chemical environment in the solvent $[36,37]$. 

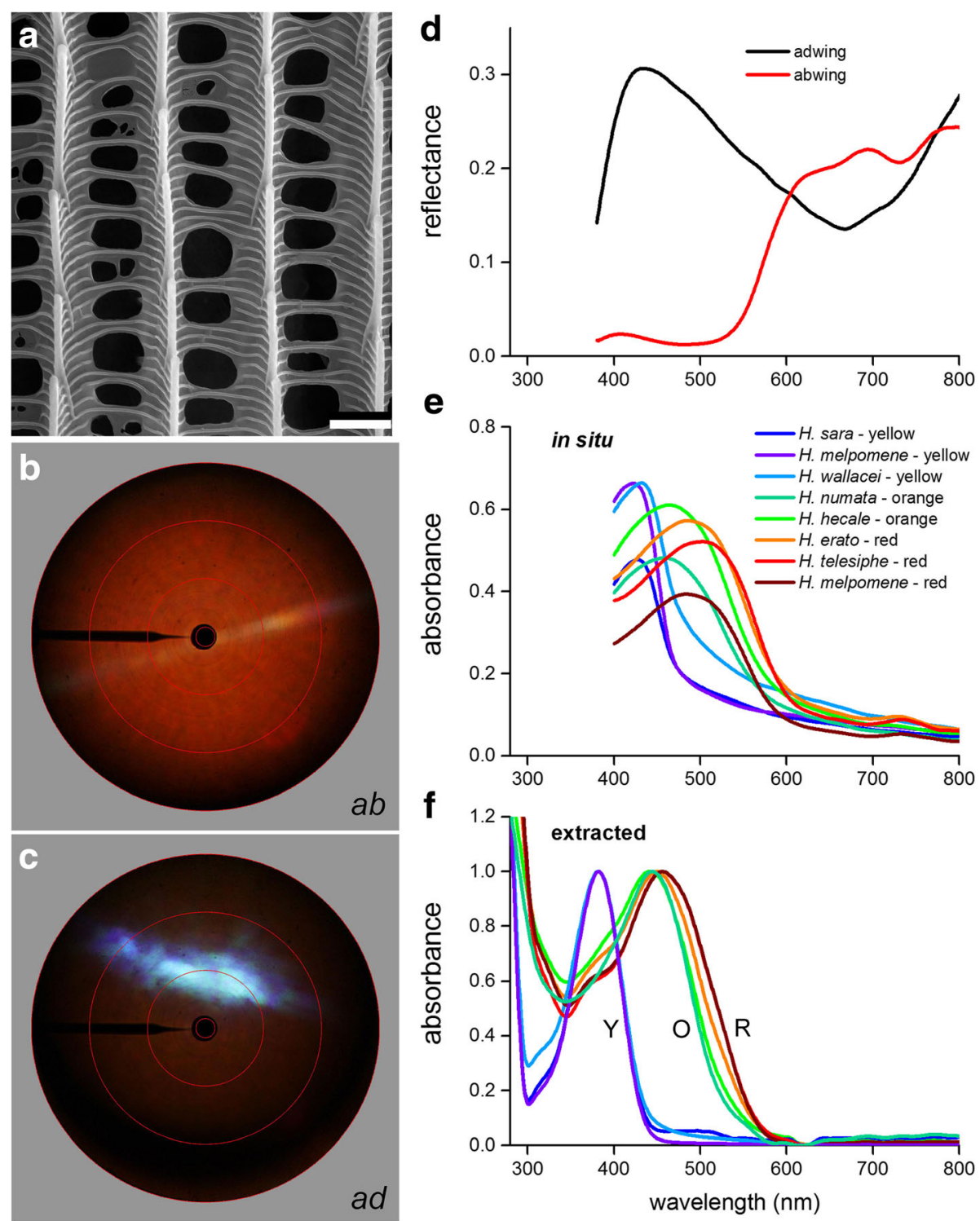

Fig. 2 Ultrastructure, spatial scattering and spectral characteristics of pigmented wing scales. a Ultrastructure of a pigmented wing scale (scale bar: $2 \mu \mathrm{m})$. b, c Imaging scatterograms of a red-colored wing scale of $\mathrm{H}$. telesiphe using local illumination on the abwing (upper) (b) and adwing (lower) (c) side. $\mathbf{d}$ Reflectance spectra of single red-colored wing scales of H. telesiphe measured from the adwing and abwing side. e Absorbance spectra of single wing scales immersed in refractive index matching fluid $(n=1.56)$. $\mathbf{f} U \mathrm{~V}$-Vis absorbance spectra of extracted yellow $(\mathrm{Y})$, orange $(\mathrm{O})$ and red (R) pigments

Most probably, the yellow (Y) pigment is 3-OHK $[10,38-41]$, while the orange $(\mathrm{O})$ and red $(\mathrm{R})$ pigments are the ommochromes xanthommatin and dihydroxanthommatin [2, 18, 32, 42, 43]. Previously, Bybee et al. [2] extracted the yellow pigment from the wings of 13 Heliconius species (eight of which overlap with the species in this study), and used mass spectrometry to demonstrate that the yellow pigment is indeed 3-OHK. We conclude that the dorsal yellow wing areas of the species depicted in Fig. 1 have scales with abundant 3-OHK, which causes the low reflectance in the (ultra)violet and the high reflectance at the longer wavelengths (Fig. 1e); the orange and red wing areas have scales that derive their color from (mixtures of) different ommochrome pigments $[2,18]$.

Curiously, the absorbance spectra of red wing scales feature a minor peak at $\sim 720 \mathrm{~nm}$ (Fig. 2e), which is absent in the absorbance spectra of the extractions (Fig. 2f). The peak indicates trace amounts of (presumably) bile pigment, responsible for the quite noticeable dip in the reflectance spectra of Fig. 1e $(\# 1,2)[44,45]$. 
Spatial and spectral reflection properties of structuralcolored scales

Scanning electron microscopy of scales of the white and blue wing areas (Fig. 1a-d, \#6-8) showed ultrastructures similar to that of the red scales (Figs. 2a and 3a, e). Interestingly, upon isolation of the scales of the white wing area of $\mathrm{H}$. telesiphe it appeared that they were in fact blue, similar to those encountered in the closely related nymphaline butterflies [18]. Applying imaging scatterometry with the narrow-aperture light beam incident on the abwing (upper) side of a blue scale produced a diffuse, bluish scatterogram with a prominent line (Fig. 3b). Illuminating the adwing (lower) side of the scale yielded a scatterogram with a locally restricted, blue spot (Fig. 3c). The latter is due to the lower lamina acting as an approximately flat thin film, with thickness $\sim 220 \mathrm{~nm}$, as follows from the reflectance spectrum of the adwing side of the scale, measured with a microspectrophotometer (MSP) (Fig. 3d, c.f. Fig. 2d). The reflectance spectrum of the abwing side is very similar (Fig. 3d), meaning that the lower lamina of the thin film principally determines the scale color. The scales are unpigmented (not shown) and therefore most of the light flux entering from the normal, abwing side will pass the upper lamina and will then be partly reflected by the lower lamina. Most of that reflected light will pass the upper lamina again, where it will be diffused by the upper lamina structures, thus causing the bluish diffused scatterogram of Fig. 3b [18]; the line in Fig. $3 \mathrm{~b}$ again results from the ridges acting as a grating.

Both cover and ground scales in the white band of Fig. 1a are blue, but as they form a stack of partly overlapping scales in situ, the multiple scattering causes an elevated reflectance spectrum with a hump in the blue wavelength range and thus a whitish color (Fig. 1a,f, \#8). We encountered the identical situation in a number of nymphaline butterflies [18]. Interestingly, blue cover scales overlapping black ground scales cause the distinct blue wing areas of the dorsal hindwings of the Doris Longwing, subspecies Heliconius doris doris (Fig. 1d), again very similar as occurs in the nymphaline butterflies [18].

We initially expected that this would be also the case in the iridescent blue wing areas of the Sara Longwing, $H$. sara (Fig. 1e, \#7). Scanning electron microscopy of the scales yielded in principle a similar scale structure as that of the blue scales of $H$. telesiphe, but closer inspection showed that the wing scale ridges are folded into a rather primitive multilayer of 2 to 3 layers (Fig. 3e). The scatterogram of the blue wing scales of $H$. sara illuminated from the abwing side is again line-shaped, similar to $H$. telesiphe due to diffraction by the ridges, but the widefield, diffused blue scattering encountered in $H$. telesiphe is absent in the H. sara scale (Fig. 3f). The reason was revealed when inspecting the adwing side of the $H$. sara scale, which appeared to have a purplish instead of a blue color. The scatterogram of the adwing side is again spatially restricted (Fig. 3g), as in H. telesiphe (Fig. 3c), confirming that the lower lamina also here acts as a thin film reflector. However, the reflectance spectra measured from both scale sides with the microspectrophotometer (Fig. 3e) show that the physical mechanisms underlying the reflection properties of both sides of the $H$. sara scale must be quite different (Fig. 3h). The adwing reflectance spectrum indicates a thin film reflector of thickness $\sim 180 \mathrm{~nm}$ in the lower lamina, causing a purplish instead of a bluish color. Furthermore, the blue H. sara scales appeared to contain melanin pigment (Additional file 1: Figure S2), which effectively suppresses incident light from the abwing side that is reflected by the lower lamina. We conclude that the abwing reflectance spectrum, peaking at $\sim 500 \mathrm{~nm}$, is due to the multilayered ridges, similar to the case of the well-known Morpho butterflies, where the intense blue coloration is caused by scales with multilayered ridges with up to 10 layers $[25,34,46]$.

\section{Color tuning by pigment filtering}

We encountered a modification of the simple blue scales of $\mathrm{H}$. telesiphe in the Doris Longwing subspecies, $\mathrm{H}$. doris doris and $H$. doris viridis. Heliconius doris is the only species exhibiting color polymorphism within populations $[24,47]$. In the investigated butterflies, both subspecies have dorsal forewings with prominent yellow patches. The reflectance spectrum of the yellow wing area, measured with the bifurcated probe (Fig. 4, \#1), demonstrates that the yellow color is determined by 3OHK, which strongly absorbs scattered light in the short-wavelength range (Fig. 2f). The difference between the two $H$. doris subspecies is that the dorsal hindwings are blue in $H$. $d$. doris (Fig. 1d) and green in $H$. d. viridis (Fig. 4). As noted above, the blue scales of $H$. d. doris are unpigmented, but in the case of $H$. d. viridis the green scales clearly contain a substantial amount of 3OHK. The reflected and scattered blue light, when spectrally filtered by $3-\mathrm{OHK}$, results in the greenish color (Fig. 4, \#2). Indeed, after extraction of the 3-OHK pigment using acidified methanol (see above), the greencolored wing patch becomes blue (dotted line, Fig. 5).

\section{Phylogenetic relationships}

We investigated the distribution of each of the seven types of colored scales identified in Heliconius in relation to the species' phylogeny. The systematics of the genus Heliconius has been thoroughly studied due to their mimicry and rapid speciation [48-50]. We used the phylogeny of Heliconius proposed by Kozak et al. [24] to map the investigated pigmentary and structural traits. 

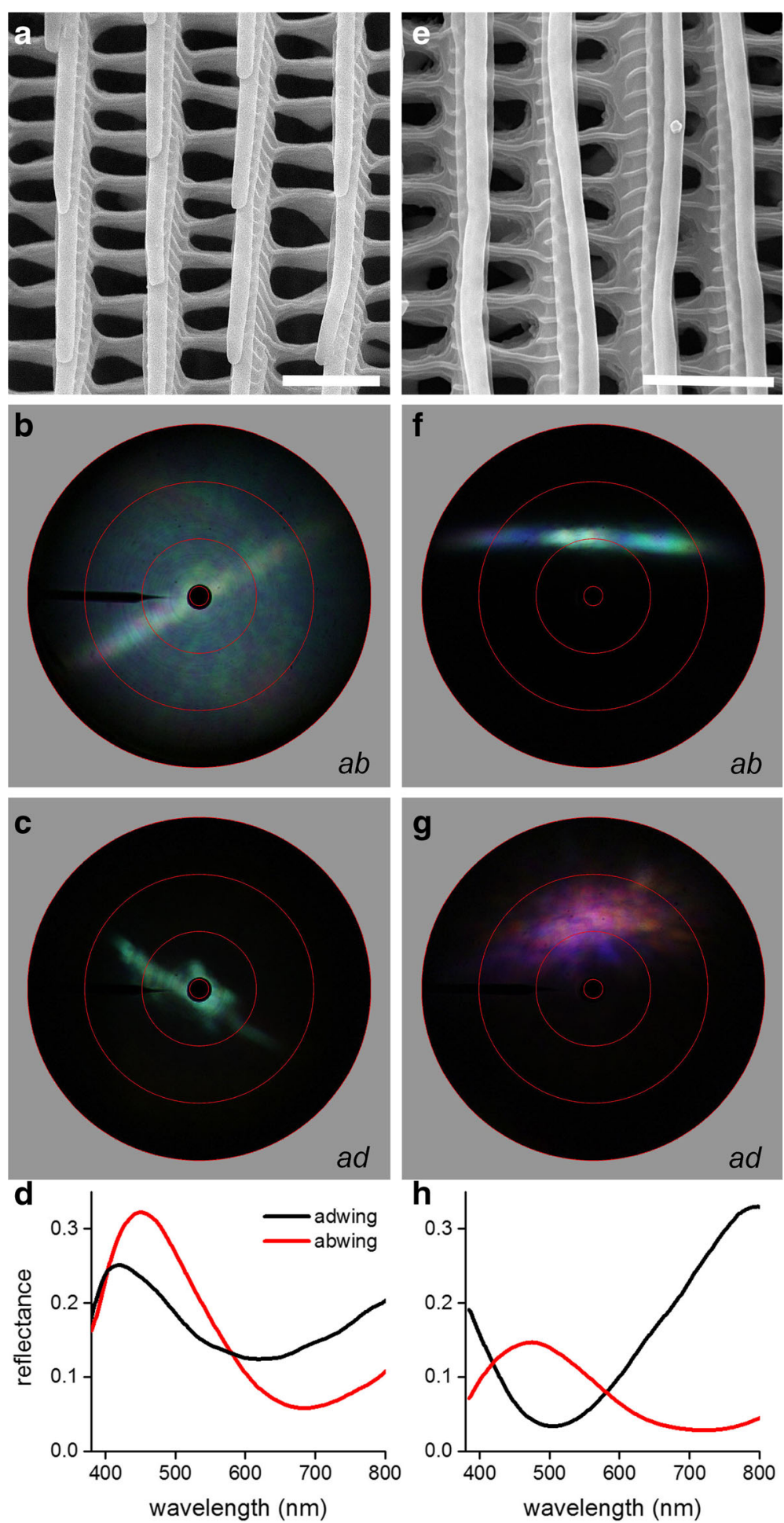

Fig. 3 Optical characteristics of the structural colored scales of $H$. telesiphe (a-d) and $H$. sara (e-h). a, e Scanning electron micrographs of a scale from the white area of $H$. telesiphe and a scale from the blue wing area of $H$. sara (scale bars: $2 \mu \mathrm{m}$ ). $\mathbf{b} \mathbf{- g}$ Imaging scatterograms of the abwing (upper) (b, f) and adwing (lower) (c, $\mathbf{g})$ sides. $\mathbf{d}, \mathbf{h}$ Reflectance spectra from both sides of the wing scale

Additional file 1: Figure S4, S5 and Table S1 list six kinds of scales whose colors derive from either the presence of pigments (yellow, orange/red) or the scale (nano)structures (simple scales or multilayers in the ridges) for the investigated heliconians. Each trait represents a distinct scale type present on the wings of that species; species can have more 


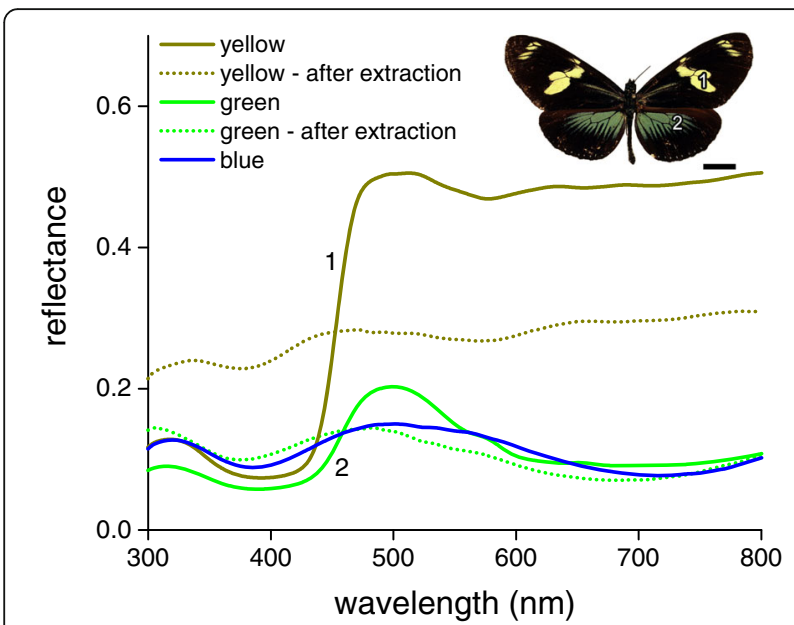

Fig. 4 Spectral tuning in $\mathrm{H}$. doris. The blue areas of the dorsal hindwings of the blue morph of $\boldsymbol{H}$. d. doris (see Fig. 1d) are green in this specimen of H. d. viridis (inset; scale bar: $1 \mathrm{~cm}$ ). The solid lines line show the reflectance spectra for the various morphs, while the dotted lines show the reflectance spectra measured after extraction of the pigment using acidified methanol (see Methods and Additional file 1: Fig. S2)

than one type of colored scale. Previously, Heliconius scales have been classified according to ultrastructure and pigmentation as Type I (yellow or white), Type II (melanic) or Type III (orange or red) [32, 51-53]. Here we classify all three types as simple scales either with or without pigment, and to this classification we identify a new scale type with multilayered ridges.

All species possess melanin, which is therefore not included in either Additional file 1: Table S1 or Figure S4 and S5. Virtually all species have one or more of the three identified pigments in their brightly colored bars, with 3-OHK being the most abundant and its derivatives xanthommatin and/or dihydroxanthommatin being less frequent (Additional file 1: Figure S4A). Maximum likelihood ancestral state reconstruction suggests that scales with 3-OHK were present at the base of the genus Heliconius. Scales with xanthommatin and/or dihydroxanthommatin were also likely present at the base of both Heliconius and Eueides, although the data are less conclusive. The latter is likely an artefact of our sampling method, which sampled pigments from large colored wing patches due to the difficulty of pigment extractions from small wing patches. Several Heliconius species (H. cydno, H. sapho, H. charithonia) have minor red patches near the base of their hindwings that are too small for extraction. Thus dihydroxanthommatin is likely to be widely distributed across the genus.

The scale types whose colors derive solely from structural parameters (Additional file 1: Figure S4B and S5, right) are also highly variable among the various species and are not conserved within sub-clades, although no real trend in the data is apparent. Ancestral state reconstruction suggests a 50\% likelihood that stacked simple-blue scales (white) as well as scales with multilayered ridge reflectors (blue) were present at the base of both the Heliconius and Eueides genera. Simple-blue scales covering black ground scales seem to be less common than the "white" scales, which result from stacking blue scales (Additional file 1: Figure S5, right). The green scales are the most interesting of all, as they appear only in $H$. doris viridis, which suggests that a new mechanism for uptaking 3-OHK in simple-blue scales must have evolved along the branch leading to H. doris (Additional file 1: Figure S5, right).

\section{Discussion}

The coloration toolkit of longwing butterflies

The wings of longwing butterflies have pigmentary as well as structural coloration. Pigments expressed in butterfly wing scales absorb scattered light in the shortwavelength range and thus typically act as long-pass spectral filters. We have identified three distinct absorption spectra associated with the pigments from colored wing patches (Fig. 2e, f): $i$ ) yellow 3-OHK, ii) orange xanthommatin and iii) red dihydroxanthommatin. 3OHK is the precursor in the biosynthetic pathway by which xanthommatin and ultimately dihydroxanthommatin are synthesized within the developing scale cells $[37,41,54]$. While $3-\mathrm{OHK}$ is mostly pure within the scales, it seems that the orange-red scale colors are finely tuned by mixing xanthommatin and dihydroxanthommatin (Fig. 2e). The absorption properties of the various pigments embedded in scales differ substantially from the pigments when extracted, probably because of the chemical environment of the scale [37]. The pigments produce the bright, localized color patches on Heliconius wings, and the velvet black frame of melanincontaining scales further increases the contrast of these patches (Fig. 1).

Structure-based colors are either due to the reflection of light at the scale's lower lamina acting as a thin film (simple scales, e.g. in H. telesiphe, Fig. 3a-d) or at the ridge-reflector scales with multilayered ridges (e.g. in $H$. sara, Fig. 3e-h). When the simple scales are pigmented, incident light at the upper lamina will be scattered and spectrally filtered by the pigment present in the ridges (see above). The light fraction reaching the lower lamina will after reflection at the thin film be again spectrally filtered and hence contribute little to the overall scale reflectance. Consequently, the reflectance spectra of pigmented scales of the adwing or abwing side can be very different (Fig. 2d) [18]. Spectral filtering is absent in unpigmented scales where the lower lamina acts as a prominent thin film. The reflectance spectra measured with abwing (upper) and adwing (lower) illumination then are similar and feature typical thin film photonics 


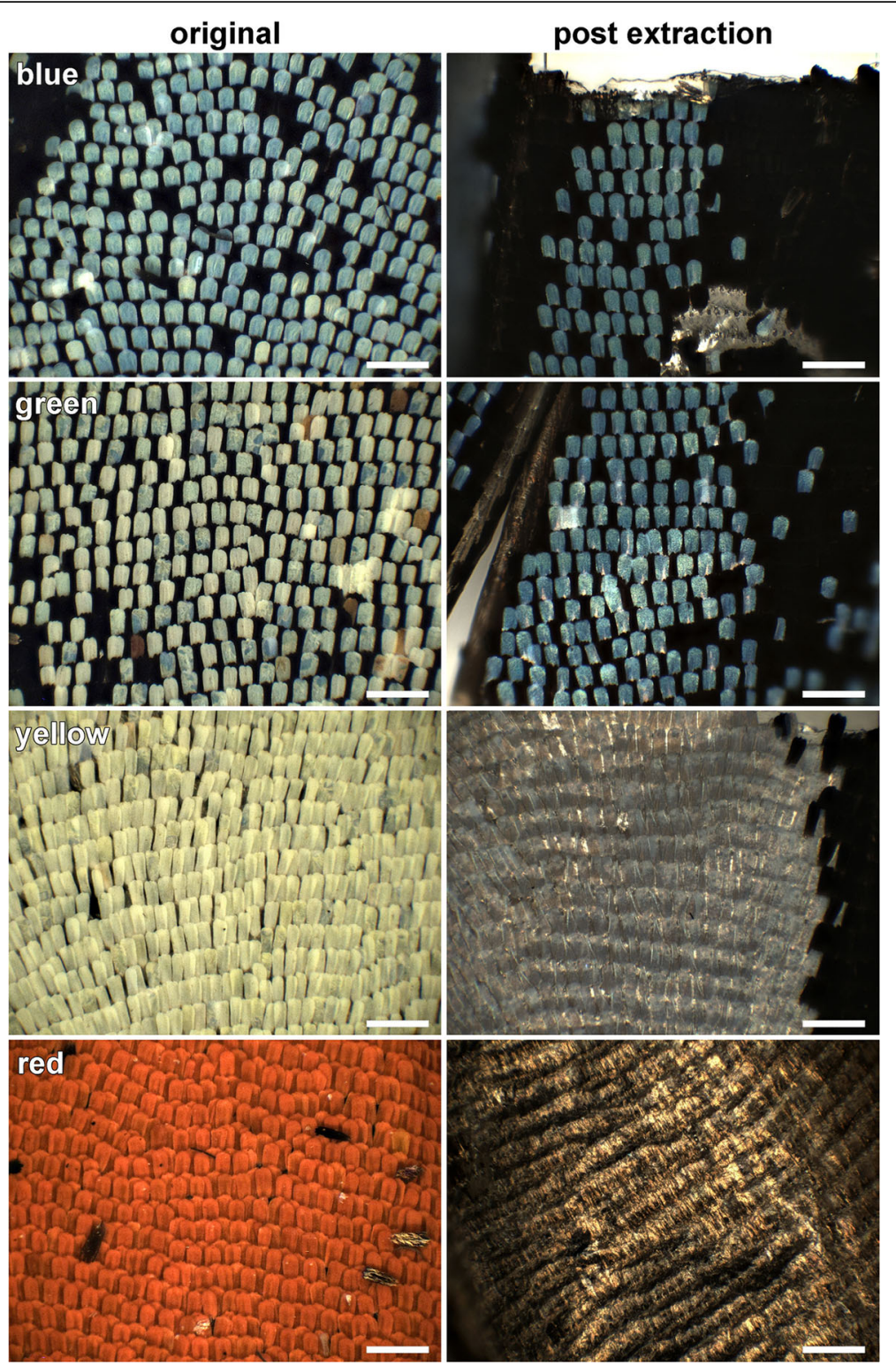

Fig. 5 Pigment extraction. Wing scale lattice of different colored wing patches in H. doris morphs before (left) and after (right) extraction in acidified methanol (see Materials and Methods). While the blue patch remains blue colored, the green patch becomes blue and the yellow and red patches uncolored, confirming the presence of pigment in these scales. Scale bars: $200 \mu \mathrm{m}$

$[18,55]$. This is the case for the blue scales that determine the color in the blue and white wing patches (Fig. 3d).

On butterfly wings, scales are stacked like shingles on a roof so that a considerable fraction of the incident light passes the cover scales and reaches the underlying ground scales. These scales thus can also contribute to the wing reflectance. As the reflectance of the melanized black ground scales is negligible, blue scales overlapping black ground scales create a blue wing color as seen in $H$. doris doris, whereas blue scales stacked on top of each other on the wing substrate yield a desaturated white color as in H. telesiphe (Fig. 1a). The reflectance spectra of the white areas having peaks in the blue wavelength range (Fig. 1f, \#8) reveal the presence of scales with blue thin-film reflectors.

Sweeney et al. [56] made the general statement, without further evidence, that thin-film interference creates the iridescent coloration of Heliconius butterflies, serving polarization signaling. However, here we have shown that the iridescent-blue wing scales of $H$. sara and $H$. erato are due to ridge-reflectors, as the scales have ridges folded into 2-3 overlapping layers (Fig. 3e) [57]. Together with the presence of melanin, light reflected 
from these scales is directionally scattered into a limited solid angle (Fig. 3f).

Structural colors can be enhanced or modified by pigments $[14,15,58]$. In the wing scales of $H$. sara, the underlying melanin pigmentation reduces scattering of transmitted (i.e. non-reflected) light that would otherwise desaturate the color signal. In the green-morph of $H$. doris viridis, pigmentary filtering of short wavelengths by 3 -OHK occurs in blue-structural colored scales, such as those featured in the blue morph of $H$. doris doris (Figs. 4 and 5).

\section{Biological function of longwing coloration: Mimicry, speciation and sexual selection}

Longwing butterflies benefit from mimicking other unpalatable butterfly species in their local habitat, as doing so spreads the cost of educating predators. The bright color patches of Heliconius wings serve as aposematic warnings to predators, advertising the distasteful nature of the butterflies [3]. The selective advantage of advertising distastefulness has led to a series of co-mimicry complexes in Heliconius, where multiple species converge on a similar color pattern $[3,59]$. The most wellknown system is that of $H$. erato and $H$. melpomene [60-62], which have similar wing patterns in local populations but diverse patterns between geographic regions. Indeed, the two subspecies of $H$. erato and the two subspecies of the highly color-polymorphic genus $H$. doris investigated here (see also Figs. 4, 5, Additional file 1: Figure S3-S5 and Table S1) share common structural motifs but have different pigments in their wing scales. It will be interesting to investigate coloration mechanisms in light of habitat-induced pressures. Heliconius species occupy light environments that can vary in brightness by one order of magnitude (see references in Seymoure et al. [63]), and can range from closed forest understory to bright open habitats. In order for the aposematism and mimicry to be successful, the longwing butterflies must continually fine-tune their colors to warn predators of their unpalatability. In this context, sexual selection is important for maintaining aposematism as it helps to select for specific shades of colors rather than the general appearance of the butterfly [4]. This has been recently demonstrated using behavioral tests where $H$. erato, given a choice of butterfly paper models that were identical in pattern, were shown to prefer 3-OHK yellow to a shade of yellow with a reflectance spectrum similar to that of co-mimics in the genus Eueides. By contrast, avian predators attacked butterfly paper models differing in shades of yellow at an equal rate under field conditions [64].

Sharing similar wing patterns brings about an additional selective pressure, namely that individuals must be able to differentiate conspecifics from heterospecifics in order to successfully reproduce. Heliconius butterflies have experienced a duplication of the UV-sensitive visual opsin pigment, which, along with the simultaneous expression of the UV-absorbing yellow pigment 3-OHK in the wings, has expanded the set of yellow colors that can in principle be distinguished by these butterflies $[2,10,64]$. Furthermore, the eyes of $H$. erato contain heterogeneouslyexpressed filtering pigments that enhance discrimination in the red wavelength range [65] via their filtering of a greenabsorbing rhodopsin in some ommatidia, resulting in a redsensitive receptor [66]. This is in contrast to butterflies like the lycaenid Polyommatus icarus, which uses a duplicated blue opsin to see green [67], or the papilionid Papilio xuthus, which uses both duplicated green opsins and filter pigments to produce red receptors [68].

The gene (optix) that globally regulates the development of red-pigmented Drosophila eyes has been re-deployed to control the expression of dihydroxanthommatin in the wings of Heliconius [23]. Optix is not, however, directly involved in regulating the expression of red filtering pigments in Heliconius eyes [69]. The evolutionary radiation of Heliconius butterflies is underlined by the diversification of pigments in the genus and the concurrent expansion of visual pigments that enhance the discrimination of similar colors [70].

Studies into the genetic processes determining coloration in Heliconius have indicated that surprisingly few genetic loci are involved in color pattern determination [23, 41, 62, 71]. Longwing species have undergone a remarkable adaptive radiation [41], driven in part by the color-pattern divergence between closely related species and mimicry-related color-pattern convergence between distantly related species [71]. A single gene controlling the expression of different wing patterns has also been found in Papilio dardanus [72, 73], which suggests that the genetic basis underlying diversity of wing patterns and species may be less complex than originally thought. It is truly remarkable that the observed vast diversity in species can be brought about by the convergence toward similar color patterns and relatively simple genetic mechanisms that control pigment expression together with a small number of optical mechanisms.

\section{Conclusion}

In conclusion, we have unraveled the physical and chemical origins of the wing scale coloration in seven kinds of colored scales in longwing butterflies. Our results indicate that a limited set of pigments and structural mechanisms produce the diverse brightly colored wing patches observed in this group. Multiple mechanisms are often combined in non-trivial ways on both a species- and subspecies-specific basis to produce new or unique colors indicating high rates of 'plasticity' that might be driven by various biological pressures, e.g. mimicry and sexual selection. 


\section{Additional file}

Additional file 1: Figures S1-S6 and Table S1. (DOCX $2441 \mathrm{~kb}$ )

\section{Acknowledgements}

We thank Hein Leertouwer for invaluable help and technical support and Jennifer Briner for assistance with specimen collection.

\section{Funding}

This research was supported by the Ambizione program of the Swiss National Science Foundation (168223, to BDW), the National Centre of Competence in Research Bio-Inspired Materials (to BDW), the U.S. National Science Foundation (IOS-1656260, to ADB) and the Air Force Office of Scientific Research/European Office of Aerospace Research and Development AFOSR/EOARD (FA9550-15-10068, to DGS).

\section{Availability of data and materials}

All data generated or analysed during this study are included in this published article and its additional files.

\section{Authors' contributions}

Study design: BDW, DGS; performed measurements: AJMV, BDW; analyzed measurements: AJMV, BDW, ADB, DGS; wrote the manuscript: BDW, DGS, $A D B$; all authors gave final approval for publication.

\section{Ethics approval and consent to participate} Not applicable.

\section{Competing interests}

The authors declare no competing or financial interests.

\section{Publisher's Note}

Springer Nature remains neutral with regard to jurisdictional claims in published maps and institutional affiliations.

\section{Author details}

${ }^{1}$ Computational Physics, Zernike Institute for Advanced Materials, University of Groningen, Nijenborgh 4, NL-9747AG Groningen, The Netherlands. ${ }^{2}$ Adolphe Merkle Institute, University of Fribourg, Chemin des Verdiers 4, $\mathrm{CH}-1700$ Fribourg, Switzerland. ${ }^{3}$ Department of Ecology and Evolutionary Biology, University of California, Irvine, CA 92697, USA.

Received: 26 May 2017 Accepted: 15 November 2017

Published online: 21 November 2017

\section{References}

1. Mason CW. Structural colors in insects. 1. J Phys Chem. 1926;30:383-95.

2. Bybee SM, Yuan F, Ramstetter MD, Llorente-Bousquets J, Reed RD, Osorio D, Briscoe AD. UV photoreceptors and UV-yellow wing pigments in Heliconius butterflies allow a color signal to serve both mimicry and intraspecific communication. Am Nat. 2012;179:38-51.

3. Mallet J, Joron M. Evolution of diversity in warning color and mimicry: polymorphisms, shifting balance, and speciation. Annu Rev Ecol Syst. 1999;30:201-33

4. Mallet J. Shift happens! Shifting balance and the evolution of diversity in warning colour and mimicry. Ecol Entomol. 2010;35:90-104.

5. Oliver JC, Robertson KA, Monteiro A. Accommodating natural and sexual selection in butterfly wing pattern evolution. Proc R Soc B. 2009;276:2369-75.

6. Michielsen K, De Raedt H, Stavenga DG. Reflectivity of the gyroid biophotonic crystals in the ventral wing scales of the green hairstreak butterfly, Callophrys rubi. J R Soc Interface. 2010;7:765-71.

7. Wilts BD, Pirih P, Arikawa K, Stavenga DG. Shiny wing scales cause spec(tac)ula camouflage of the angled sunbeam butterfly, Curetis acuta. Biol J Linn Soc. 2013;109:279-89.

8. Mallet J, Beltran M, Neukirchen W, Linares M. Natural hybridization in heliconiine butterflies: the species boundary as a continuum. BMC Evol Biol. 2007;7:28.

9. Hines HM, Counterman BA, Papa R, Albuquerque de Moura P, Cardoso MZ, Linares M, Mallet J, Reed RD, Jiggins CD, Kronforst MR, McMillan WO. Wing patterning gene redefines the mimetic history of Heliconius butterflies. Proc Natl Acad Sci U S A. 2011:108:19666-71.

10. Briscoe AD, Bybee SM, Bernard GD, Yuan F, Sison-Mangus MP, Reed RD, Warren AD, Llorente-Bousquets J, Chiao CC. Positive selection of a duplicated UV-sensitive visual pigment coincides with wing pigment evolution in Heliconius butterflies. Proc Natl Acad Sci U S A. 2010;107:3628-33.

11. Estrada C, Jiggins CD. Interspecific sexual attraction because of convergence in warning colouration: is there a conflict between natural and sexual selection in mimetic species? J Evol Biol. 2008;21:749-60.

12. Shawkey MD, Morehouse NI, Vukusic P. A protean palette: colour materials and mixing in birds and butterflies. J R Soc Interface. 2009;6(Suppl 2):S221-31.

13. Kinoshita S, Yoshioka S, Miyazaki J. Physics of structural colors. Rep Prog Phys. 2008;71:076401.

14. Wilts BD, Wijnen B, Leertouwer HL, Steiner U, Stavenga DG. Extreme refractive index wing scale beads containing dense pterin pigments cause the bright colors in pierid butterflies. Adv Opt Mat. 2017;5:1600879.

15. Wilts BD, Matsushita A, Arikawa K, Stavenga DG. Spectrally tuned structural and pigmentary coloration of birdwing butterfly wing scales. J R Soc Interface. 2015;12:20150717.

16. Stavenga DG, Stowe S, Siebke K, Zeil J, Arikawa K. Butterfly wing colours: scale beads make white pierid wings brighter. Proc R Soc B. 2004;271:1577-84.

17. Morehouse NI, Vukusic P, Rutowski R. Pterin pigment granules are responsible for both broadband light scattering and wavelength selective absorption in the wing scales of pierid butterflies. Proc R Soc B. 2007;274:359-66.

18. Stavenga DG, Leertouwer $\mathrm{HL}$, Wilts $\mathrm{BD}$. Coloration principles of nymphaline butterflies - thin films, melanin, ommochromes and wing scale stacking. J Exp Biol. 2014;217:2171-80.

19. Finkbeiner $\mathrm{SD}$, Briscoe $A D$, Reed $\mathrm{RD}$. Warning signals are seductive: relative contributions of color and pattern to predator avoidance and mate attraction in Heliconius butterflies. Evolution. 2014:68:3410-20.

20. Dell'Aglio DD, Stevens M, Jiggins CD. Avoidance of an aposematically coloured butterfly by wild birds in a tropical forest. Ecol Entomol. 2016; 41:627-32.

21. Heliconius Genome Consortium. Butterfly genome reveals promiscuous exchange of mimicry adaptations among species. Nature. 2012;487:94-8.

22. Kronforst MR, Papa R. The functional basis of wing patterning in Heliconius butterflies: the molecules behind mimicry. Genetics. 2015;200:1-19.

23. Reed RD, Papa R, Martin A, Hines HM, Counterman BA, Pardo-Diaz C, Jiggins CD, Chamberlain NL, Kronforst MR, Chen R, Halder G, Nijhout HF, McMillan WO. Optix drives the repeated convergent evolution of butterfly wing pattern mimicry. Science. 2011;333:1137-41.

24. Kozak KM, Wahlberg N, Neild AF, Dasmahapatra KK, Mallet J, Jiggins CD. Multilocus species trees show the recent adaptive radiation of the mimetic Heliconius butterflies. Syst Biol. 2015;64:505-24

25. Stavenga DG, Leertouwer $\mathrm{HL}$, Pirih $\mathrm{P}$, Wehling MF. Imaging scatterometry of butterfly wing scales. Opt Express. 2009;17:193-202.

26. Wilts BD, Michielsen K, De Raedt H, Stavenga DG. Hemispherical Brillouin zone imaging of a diamond-type biological photonic crystal. J R Soc Interface. 2012:9:1609-14.

27. Leertouwer HL, Wilts BD, Stavenga DG. Refractive index and dispersion of butterfly chitin and bird keratin measured by polarizing interference microscopy. Opt Express. 2011;19:24061-6.

28. Maddison WP. Mesquite: a modular system for evolutionary analysis. Evolution. 2008:62:1103-18

29. Wijnen B, Leertouwer HL, Stavenga DG. Colors and pterin pigmentation of pierid butterfly wings. J Insect Physiol. 2007;53:1206-17.

30. Wilts BD, Pirih P, Stavenga DG. Spectral reflectance properties of iridescent pierid butterfly wings. J Comp Physiol A. 2011;197:693-702.

31. Ghiradella $\mathrm{H}$. Insect cuticular surface modifications: scales and other structural formations. Adv Insect Physiol. 2010;38:135-80.

32. Gilbert LE, Forrest HS, Schultz TD, Harvey DJ. Correlations of ultrastructure and pigmentation suggest how genes control development of wing scales of Heliconius butterflies. J Res Lepid. 1988;26:141-60.

33. Wilts BD, Trzeciak TM, Vukusic P, Stavenga DG, Papiliochrome II. Pigment reduces the angle-dependency of structural wing colouration in nireus group papilionids. J Exp Biol. 2012;215:796-805.

34. Vukusic P, Stavenga DG. Physical methods for investigating structural colours in biological systems. J R Soc Interface. 2009:6:\$133-48.

35. Stavenga DG. Thin film and multilayer optics cause structural colors of many insects and birds. Mater Today Proc. 2014:1S:109-21.

36. Krasovitskii BM, Bolotin BM: Organic luminescent materials: Wiley-VCH; 1988 
37. Nijhout H. Ommochrome pigmentation of the linea and rosa seasonal forms of Precis coenia (Lepidoptera: Nymphalidae). Arch Insect Biochem Physiol. 1997:36:215-22.

38. Umebachi Y. Papiliochrome, a new pigment group of butterfly. Zool Sci. 1985;2:163-74

39. Tokuyama T, Senoh S, Sakan T, Brown K, Witkop B. The photoreduction of kynurenic acid to kynurenine yellow and the occurrence of 3-hydroxy-Lkynurenine in butterflies. J Am Chem Soc. 1967;89:1017-21.

40. Brown KS. Chemotaxonomy and chemomimicry: the case of 3-hydroxykynurenine. Syst Zool. 1967;16:213-6.

41. Reed RD, McMillan WO, Nagy LM. Gene expression underlying adaptive variation in Heliconius wing patterns: non-modular regulation of overlapping cinnabar and vermilion prepatterns. Proc R Soc B. 2008;275:37-45.

42. Ferguson LC, Jiggins CD. Shared and divergent expression domains on mimetic Heliconius wings. Evol Dev. 2009;11:498-512.

43. Hines HM, Papa R, Ruiz M, Papanicolaou A, Wang C, Nijhout HF, McMillan WO, Reed RD. Transcriptome analysis reveals novel patterning and pigmentation genes underlying Heliconius butterfly wing pattern variation. BMC Genomics. 2012;13:288.

44. Rothschild M, Mummery R. Carotenoids and bile pigments in danaid and swallowtail butterflies. Biol J Linn Soc. 1985:24:1-14.

45. Stavenga DG, Giraldo MA, Leertouwer HL. Butterfly wing colors: glass scales of Graphium sarpedon cause polarized iridescence and enhance blue/green pigment coloration of the wing membrane. J Exp Biol. 2010;213:1731-9.

46. Kinoshita S: Structural colors in the realm of nature: Singapore: world scientific 2008.

47. DeVries P. The butterflies of Costa Rica and their natural history: Papilionidae, Pieridae, Nymphalidae. Princeton University Press: Princeton; 1987.

48. Brower AVZ. Phylogeny of Heliconius butterflies inferred from mitochondrial DNA sequences (Lepidoptera: Nymphalidae). Mol Phylogenet Evol. 1994;3: 159-74.

49. Brower AV, Egan MG. Cladistic analysis of Heliconius butterflies and relatives (Nymphalidae: Heliconiiti): a revised phylogenetic position for Eueides based on sequences from mtDNA and a nuclear gene. Proc R Soc B. 1997;264: 969-77.

50. Beltrán M, Jiggins CD, Brower AV, Bermingham E, Mallet J. Do pollen feeding pupal-mating and larval gregariousness have a single origin in Heliconius butterflies? Inferences from multilocus DNA sequence data. Biol J Linn Soc 2007;92:221-39.

51. Janssen JM, Monteiro A, Brakefield PM. Correlations between scale structure and pigmentation in butterfly wings. Evol Dev. 2001;3:415-23.

52. Aymone $A B$, Valente $V$, de Araújo $A M$. Ultrastructure and morphogenesis of the wing scales in Heliconius erato phyllis (Lepidoptera: Nymphalidae): what silvery/brownish surfaces can tell us about the development of color patterning? Arthropod Struct Dev. 2013;42:349-59.

53. Jiggins CD. The Ecology and Evolution of Heliconius Butterflies: A Passion for Diversity: Oxford. UK: Oxford University Press; 2017.

54. Koch PB. Production of $\left[{ }^{14} \mathrm{C}\right]$-labeled 3-hydroxy-L-kynurenine in a butterfly, Heliconius charitonia L. (Heliconidae), and precursor studies in butterfly wing ommatins. Pigment Cell Res. 1993;6:85-90.

55. Wasik BR, Liew SF, Lilien DA, Dinwiddie AJ, Noh H, Cao H, Monteiro A. Artificial selection for structural color on butterfly wings and comparison with natural evolution. Proc Natl Acad Sci U S A. 2014;111:12109-14.

56. Sweeney A, Jiggins $C$, Johnsen S. Polarized light as a butterfly mating signal. Nature. 2003:423:31-2

57. Kinoshita S, Yoshioka S, Fujii Y, Osanai M. Photophysics of structural color in the Morpho butterflies. Forma. 2002;17:103-21.

58. Wilts BD, IJbema N, Stavenga DG. Pigmentary and photonic coloration mechanisms reveal taxonomic relationships of the Cattlehearts (Lepidoptera: Papilionidae: Parides). BMC Evol Biol. 2014;14:160.

59. Counterman BA, Araujo-Perez F, Hines HM, Baxter SW, Morrison CM, Lindstrom DP, Papa R, Ferguson L, Joron M, Ffrench-Constant RH, Smith CP, Nielsen DM, Chen R, Jiggins CD, Reed RD, Halder G, Mallet J, McMillan WO. Genomic hotspots for adaptation: the population genetics of Mullerian mimicry in Heliconius erato. PLoS Genet. 2010;6:e1000796.

60. Mallet J. The genetics of warning colour in Peruvian hybrid zones of Heliconius erato and H. melpomene. Proc R Soc B. 1989;236:163-85.

61. Mallet J, Gilbert LE. Why are there so many mimicry rings - correlations between habitat, behavior and mimicry in Heliconius butterflies. Biol J Linn Soc. 1995:55:159-80.
62. Baxter SW, Papa R, Chamberlain N, Humphray SJ, Joron M, Morrison C, Ffrench-constant $\mathrm{RH}$, WO MM, Jiggins CD. Convergent evolution in the genetic basis of Mullerian mimicry in Heliconius butterflies. Genetics. 2008; 180:1567-77.

63. Seymoure BM, McMillan WO, Rutowski R. Peripheral eye dimensions in longwing (Heliconius) butterflies vary with body size and sex but not light environment nor mimicry ring. J Res Lepid. 2015;48:83-92.

64. Finkbeiner SD, Fishman DA, Osorio D, Briscoe AD. Ultraviolet and yellow reflectance but not fluorescence is important for visual discrimination of conspecifics by Heliconius erato. J Exp Biol. 2017;220:1267-76.

65. Zaccardi G, Kelber A, Sison-Mangus MP, Briscoe AD. Color discrimination in the red range with only one long-wavelength sensitive opsin. J Exp Biol. 2006;209:1944-55.

66. McCulloch KJ, Osorio D, Briscoe AD. Sexual dimorphism in the compound eye of Heliconius erato: a nymphalid butterfly with at least five spectral classes of photoreceptor. J Exp Biol. 2016;219:2377-87.

67. Sison-Mangus MP, Briscoe AD, Zaccardi G, Knuttel H, Kelber A. The lycaenid butterfly Polyommatus icarus uses a duplicated blue opsin to see green. J Exp Biol. 2008;211:361-9.

68. Arikawa K, Mizuno S, Kinoshita M, Stavenga DG. Coexpression of two visual pigments in a photoreceptor causes an abnormally broad spectral sensitivity in the eye of the butterfly Papilio xuthus. J Neurosci. 2003:23:4527-32.

69. Martin A, McCulloch KJ, Patel NH, Briscoe AD, Gilbert LE, Reed RD. Multiple recent co-options of Optix associated with novel traits in adaptive butterfly wing radiations. EvoDevo. 2014;5:7.

70. McCulloch KJ, Yuan F, Zhen Y, Aardema ML, Smith G, Llorente-Bousquets J, Andolfatto $P$, Briscoe AD. Sexual dimorphism and retinal mosaic diversification following the evolution of a violet receptor in butterflies. Mol Biol Evol. 2017;34:2271-84

71. Joron M, Papa R, Beltrán M, Chamberlain N, Mavárez J, Baxter S, Abanto M, Bermingham E, Humphray SJ, Rogers J. A conserved supergene locus controls colour pattern diversity in Heliconius butterflies. PLoS Biol. 2006;4:e303.

72. Thompson MJ, Timmermans MJ, Jiggins CD, Vogler AP. The evolutionary genetics of highly divergent alleles of the mimicry locus in Papilio dardanus. BMC Evol Biol. 2014;14:140.

73. Clark R, Vogler AP. A phylogenetic framework for wing pattern evolution in the mimetic mocker swallowtail Papilio dardanus. Mol Ecol. 2009:18:3872-84.

\section{Submit your next manuscript to BioMed Central and we will help you at every step:}

- We accept pre-submission inquiries

- Our selector tool helps you to find the most relevant journal

- We provide round the clock customer support

- Convenient online submission

- Thorough peer review

- Inclusion in PubMed and all major indexing services

- Maximum visibility for your research

Submit your manuscript at www.biomedcentral.com/submit 